\title{
Asir gradualmente la realidad: para un principio de correspondencia ontológico-epistemológico
}

\author{
Grasping gradually the reality: \\ towards an epistemological-ontological \\ correspondence principle
}

Carlos Sierra Lechuga

\section{Resumo}

Quando o homem estuda o que seja, espera que de algum modo capture o real. Este trabalho trata de esclarecer com precisão esse «modo» em que o real é o real. Os entes da razão matemática não são reais do mesmo modo que os objetos físicos do mundo ou que os entes metafísicos circunscritos na totalidade. A realidade nos resiste: não se pode dizer qualquer coisa dela e por isso é preciso distingui-la em graus. O conceito de realidade graduada não é novo (Dionísio Areopagita falava já das hierarquias no tocante ao celeste e Santo Tomás de Aquino explicitou certo graus do ser), no entanto, é urgente recuperá-lo. Nesta medida, uma realidade gradual é, frente a tudo, uma realidade estruturada. A diferença implícita entre os graus reside no modo em que estruturalmente resistem-se, tanto entre si, quanto para conosco. Para o nosso contexto, isto é pertinente para se tratar conjuntamente a ciência e a religião, pois, ainda que ambas propõem uma abordagem ao real, seu meio e fim abordá-lo é gradualmente distinto. Tal distinção evita, por sua vez, impor certas realidades e seus saberes sobre outros, pois, uma realidade estruturada, além de possuir passos consequenciais, possui graus constituintes. As implicações práticas disso são, por exemplo, distinguir os resultados físicos das conclusões 
metafísicas, ou as abordagens matemáticas ou experimentais dos místicos ou rituais; pois eles não nos resistem do mesmo modo, tal como a causa de uma aceleração (a força) e a Causa Primeira (Deus). Ainda, pois, para que nosso estudo da realidade seja coerente, deve ter-se em conta a resistência de uma realidade gradual. Investigaremos, filosoficamente, como integrar de maneira coerente os graus do saber com os graus de realidade; para nós, é crucial um Princípio de Correspondência ontológico-epistemológico (inspirados em N. Bohr). Como diria J. Maritain, teremos que distinguir para unir.

Palavras-chave: realidade, estrutura, constituição, correspondencia.

\section{Abstract}

When man studies whatever, he expects that it be real in some mode. This paper attempts to clarify precisely this «mode» in which real is real. The beings of reason in mathematics, the physical objects of the world and the metaphysical entities circumscribed in hole of totality are not real in the same mode. Reality resists us: we cannot say anything about her and therefore it is necessary to distinguish degrees. The concept of gradual reality is not new (Dionysius already talked about hierarchy on celestial and Aquinas explicit certain degrees of being), however, it is urgent to recover it. In this way, a gradual reality is, above all, a structured reality. The implied difference between degrees lies in the mode how they structurally resist, both with each other, as in front of us. This is relevant when we are trying together Science and Religion, because, even when both seek an approach to the real, their media and ends of approach are gradually different. This distinction prevents us, in turn, of imposing realities and their knowledge above others; structured reality rather than owning consequential steps, has constituent degrees. The practical implications of this are, for example, to distinguish the physical results of the metaphysical conclusions, or experimental approaches of ritual ones, because the cause of an acceleration (force) and the First Cause (God) do not resist us likewise. Thus, for a coherent study on reality, we should notice the resistence of gradual reality. We will investigate philosophically how to integrate in a coherent way the degrees of knowledge whit the degrees of reality specifying, first of all, the real kind of things manifested when we are with them, then, showing that such kind varies according to that of which is kind, thirdly, noting that this variation is due to how things are resisting, 
and finally, as with all the above and inspired by N. Bohr, proposing a crucial ontological and epistemological Principle of Correspondence. As J. Maritain suggested, we must distinguish to join.

Keywords: reality, structure, constitution, correspondence.

En cierto sentido, el científico prescinde de su fe en su trabajo, no porque esa fe pudiera entorpecer su investigación, sino porque no se relaciona directamente con su actividad científica. ${ }^{1}$

Georges Lemaitre

\section{Introducción}

Básica y primigeniamente, hay una operación realizativa de la inteligencia humana hacia aquello que no es ella misma. Cuando algo se confronta a una inteligencia, la inteligencia lo considera, básica y primigeniamente, como un algo real. ${ }^{2}$ Sea lo que sea esta realidad del algo presentado ante una inteligencia, lo cierto es que el algo no parece ilusorio, alucinación o fantasma. El modo como nos aparecen todos los algos, es en modo real. Esto lo saben bien los dedicados a la psiquiatría pues, aun para sus pacientes, incluso las

${ }_{1}$ Palabras de Georges Lemaître pronunciadas el 10 de septiembre de 1936. Citado por: ARTIGAS, M. Ciencia y fe: el origen del universo, Georges Lemaître: el padre del big-bang. Ciencia, razón y fe, Universidad de Navarra, 7 junio 1995. Disponible en: <http://www.unav.es/ cryf/georgeslemaitreelpadredelbigbang.html >. Consultado el 24 de septiembre de 2012.

${ }^{2}$ Esta idea está basada en la tesis zubiriana de la Inteligencia Sentiente. La inteligencia (no conciencia o subjetividad, etc.), como bien lo ha tratado Xavier Zubiri a lo largo de toda su obra, tiene por función primigenia no la resolución de problemas ni la facultad de juzgar sino la actividad primaria de inteligir, es decir, de aprehender de las cosas reales en tanto que reales. Así, puede decirse que mientras que los estímulos sensibles nos dicen del calor, por ejemplo, que calienta, la inteligencia nos dice, además, que es caliente, esto es, de darle a la cosa inteligida un carácter propio. La inteligencia tiene un primer momento constitutivo - en virtud del cual se llama, más bien, inteligencia sentiente- que es: la impresión de realidad. Dicho momento suyo es aquel que constituye la formalidad del contenido de lo inteligido como formalidad de realidad; lo inteligido "tiene" forma de real. La bibliografía para lo dicho es vasta, señalaré sólo algunas de referencias: La trilogía de inteligencia sentiente, particularmente el primer tomo: ZUBIRI, X., Inteligencia sentiente: inteligencia y realidad. Madrid: Alianza, 1980. Asimismo: ZUBIRI, X. El hombre: lo real y lo irreal. Madrid: Alianza, 2005. ZUBIRI, X. El hombre y la verdad. Madrid: Alianza, 2005. ZUBIRI, X. Reflexiones teológicas sobre la eucaristía. Estudios escolásticos 56 (1981) Enero-Junio, 41-59. ZUBIRI, X. Los problemas fundamentales de la metafísica occidental. Madrid: Alianza, 2008. ZUBIRI, X. Respectividad de lo real. Realitas III-IV (1976-1979), 13-43. ZUBIRI, X. ¿Qué es investigar? The Xavier Zubiri Review 7 (2005), 5-7. 
alucinaciones parecen reales. Así pues, existe una operación realizativa sobre la totalidad, donde realizar significa tan sólo que cada parte, estructura, elemento, propiedad, etc., de dicha totalidad, e incluso la totalidad misma, son para el hombre realidades, realidades que en cuanto le salen al paso, le salen -justamente- como siendo reales.

\section{Lo Real de la Realidad}

El modo en que lo inteligido es inteligido como siendo real, es también patente cuando el hombre no sólo intelige sino que investiga. ${ }^{3}$ Cuando el hombre investiga lo que sea, espera que de algún modo sea real. En efecto, lo espera, otra cuestión es saber si efectivamente es real. Un entomólogo, por ejemplo, aborda la realidad de un insecto con sus conceptos entomológicos, y la manifestación de la realidad insectiva es una manifestación real, es decir, el hombre no se cree a sí mismo como un diablillo hipotético investigador de insectos, ${ }^{4}$ no cree tampoco estar investigando meras ilusiones, no cree, además, que la entomología sea una gimnasia mental; sino que atestigua efectivamente que él es realmente un entomólogo, que el insecto investigado es real y que su abordaje entomológico realmente dice algo de la realidad insectiva. Atestigua, pues, la realidad de su investigación. Saber si en ese atestiguamiento hay convergencia entre el estudio y lo estudiado es otra cuestión - la cuestión por la verdad - pero aquí no tratamos de ello sino sólo de este modo, muy básico y primigenio, de realidad en que se desarrolla el atestiguamiento. Este atestiguamiento de la realidad me parece claramente notable en el verbo castellano "estar". Aunque posiblemente ocurra lo mismo en portugués, en castellano "estar" es mucho más fuerte que el mero "ser". Pues en castellano no nos significa lo mismo decir, por ejemplo, "yo soy vidente" que "yo estoy viendo" ${ }^{\prime}$, ambos hablan de un yo que ve, pero el modo como se ve es diferente. "Yo soy vidente" reporta una cualidad mía: la de poder ver, reporta que eventualmente puedo ver; mientras que en "yo estoy viendo" constato vivamente, actualmente, lo que de facto hago: ver aquí y ahora. El modo de

\footnotetext{
${ }^{3}$ El investigar es un caso concreto del mero inteligir.

${ }^{4}$ Cfr. los diablillos de Descartes (genio maligno), de Laplace y de Maxwell.

${ }^{5}$ Para el lector de habla inglesa: el inglés podría afirmar "I am watcher" y "I am watching", pero aquí lo que cambia no es el verbo, la acción, sino aquello que se dice del sujeto, del "I". Esto delega la carga semántica, delegando la fuerza del verbo al predicado, perdiendo la intensidad el verbo to be.
} 
ver en el primer caso es cualitativo, ser vidente me cualifica; el modo de ver en el segundo es estrictamente realizativo, estar viendo efectivamente. El sentido fuerte del estar respecto del ser radica en que cada vez que estoy lo estoy concreta, física y patentemente. Por lo tanto, pienso que el atestiguamiento de la realidad en tanto realidad, no está, como ha creído gran parte de la metafísica clásica, en el ser sino en el estar. Mas no le doy al estar peso ontológico alguno, pues no es más que una etiqueta lingüística - castellana - que me ayuda a denotar una situación concreta, la cual - esa sí - tiene peso ontológico: el básico y primigenio contacto con lo demás, contacto que nos hace patente $s u$ realidad, la realidad de eso demás. Llegamos al ser, como el mismo Santo Tomas de Aquino ya pensaba, posteriormente a un proceso de abstracción, nuestro primer contacto no es con el ser - dice - sino con aquelloque-está-siendo, el ens; ${ }^{6}$ con eso concreto cuyo acto es ser, ${ }^{7}$ pero no con el ser mismo. ${ }^{8} \mathrm{El}$ ser podrá ser lo fundamental que se quiera, pero no es lo más básico y primigenio. El primer contacto con las cosas concretas se atestigua en el estar, porque antes de percatarme del ser de las cosas me percato de que estoy con ellas y de que ellas están ahi (conmigo). "Estar" denota, pues, la situación en la que me encuentro circunscrito por algo que me aparece como siendo real. Esta diferencia que en castellano puedo hacer entre el ser y el estar me permite darle al primero toda la carga metafísica u ontológica que la tradición ha dado, pero también me da en el segundo - en el estar - una puerta de acceso para explicar lo que todos, hablemos o no castellano, patentizamos cada vez que contactamos con algo. ${ }^{9}$ Es una situación bien concreta y válida

\footnotetext{
6 "ens autem et essentia sunt quæ primo intellectu concipiuntur" tomado, como él mismo reconoce, de Avicena. AQUINO, Tomás de, De ente et essentia. Luventicus, 9 de diciembre de 2003. Disponible en <http://www.luventicus.org/articulos/03Tr002/index.html\#IIb $>$. Consultado el 13 de septiembre de 2012.

7 Pues: "est ens id cuius actus est esse", según Tomás de Aquino en su Opúsculo De natura generis, citado por ZUBIRI, X. Los problemas fundamentales de la metafísica occidental. Madrid: Alianza, 2008, p. 91.

${ }^{8}$ Incluso, en sentido estricto, esto ya era sabido por Aristóteles. El ser es primordialmente ovøía, pero no llegamos a ella hasta que no la depuramos de los demás modos de ser (de las restantes nueve categorías fundadas todas en la ovoía). Vid. Met 1028b1-8.

${ }^{9}$ En cuanto al ser, éste puede significar todo lo que la historia de la metafísica ha debatido, aunque no sea lo más básico y primigenio para una inteligencia humana. No es lo más básico y primigenio no sólo porque, como dice Tomás, haya que hacer un proceso de abstracción en las cosas para llegar al momento del acto de ser (aunque para él - si bien no es lo más básico y primigenio - sí es lo fundamental), sino por algo mucho más elemental, a saber, que existen lenguas - por ejemplo el hebreo - en las que el verbo ser (al menos en presente) no figura, y si figura no es tan tipificante como la historia de la metafísica occidental lo ha considerado. No hay en ciertas culturas, parti-
} 
para quien sea que mi lengua me permite denotar con la llana etiqueta "estar". Cuando digo, entonces, que estoy investigando, constato - incluso físicamente - que ya, hic et nunc, investigo realmente. Decir que soy investigador no implica a fortiori que lo sea ahora, pues bien pudiera ser investigador sin ahora mismo estar investigando, mientras que decir que estoy investigando implica taxativamente que soy investigador (soy investigador al menos y precisamente en ese momento en que estoy investigando). Se patentiza, pues, la realidad actual en el estar. Lo básico y primigenio de la aparición de lo real en tanto que real está precisamente en esta base primigenia del estar previo al ser. Y puesto que el ser es segundo respecto del estar, quizá el ser nos sea más familiar - ocurre que lo primero siempre es ignorado porque se da por obviado -, y entonces podremos entendernos lingüísticamente diciendo que estar es ser real en sentido fuerte. No es que en el ser no haya realidad, sino que ésta, la realidad, se patentiza - desde lo más básico y primigenio - no tanto en el ser como en el estar.

En el estar se patentiza fuertemente la realidad de aquello en lo que se está, por lo tanto también de aquel quien está y, con ambos, del estar en cuanto tal. Estar investigando supone la realidad del investigador, de lo investigado y del investigar. Al estar investigando, entonces, lo investigado es de algún modo real. Pues bien, tratemos ahora de esclarecer precisamente este "modo" en que lo real es real, para luego notar que entre modos hay considerables diferencias, pues está claro que no son del mismo modo reales los entes de razón de la matemática que los objetos físicos del mundo o que los entes metafísicos circunscritos en la totalidad. No parecen estar realmente todos del mismo modo. En efecto, ¿está la causa de una aceleración, la fuerza, al modo como lo está la Causa Primera, Dios? No; hay dos causas que no están del mismo modo. Veamos cómo estos modos requieren de la distinción de grados en los modos de estar, de ser real.

cularmente orientales, el interés por el ser porque en su cosmovisión, estructurada por y con su lenguaje, el ser no tiene un papel central y, por lo tanto, no les parece ser el sustento ontológico

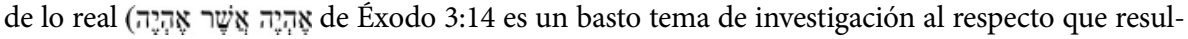
taría interesante hacer manteniendo a raya los prejuicios occidentales). El ser es, en este sentido, discutible. Pero el estar no. Es cierto que el verbo estar no aparece en muchísimas lenguas, sino tan solo en algunas romances, pero, como he dicho, no hago del estar algo metafísico, no le doy peso ontológico en sí, sólo utilizo lo que mi lengua me permite para señalar, denotar, etiquetar, una situación concreta: el contacto diario con y en el mundo, la aparición de lo que sea a una inteligencia, el atestiguamiento de lo real. Así pues, para no decir todas estas situaciones, simplemente abrevio con la palabra "estar". Mi uso del estar es, pues, pedagógico, no ontológico. Denota esa situación básica y primigenia de la aparición de lo real. 


\section{Grados de Realidad}

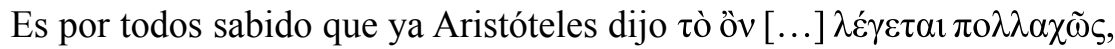
${ }^{10}$ (lo) que es se dice de muchos modos ${ }^{11}$, y nos muestra cuatro modos en que se dice que es (ser independiente - ov $\sigma^{i} \alpha$-, ser por sí mismo - $\kappa \alpha \theta^{\prime} \alpha v \tau o ́$ -, ser verdadero - $\dot{\alpha} \lambda \eta \theta \dot{\varepsilon} \varsigma-$ y ser acto - $\dot{\varepsilon} v \tau \varepsilon \lambda \dot{\varepsilon} \chi \varepsilon 1 \alpha-)$. Aunque todos ellos pudieran ser correctos, a mi parecer son insuficientes. Insuficientes al menos de cara a este trabajo, donde no tratamos propiamente del tò ôv, del "que es",

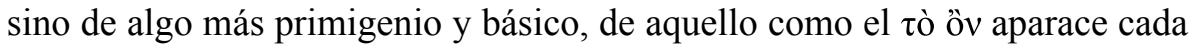
vez que uno está en lo que es. Cada vez que uno está en lo que es, esto que es - el $\tau$ ò ồ - aparece de modo real, y de lo real y sus modos nos ocupamos aquí. En cualquiera de los varios modos ( $\pi \mathrm{o} \lambda \lambda \alpha \chi \tilde{\omega} \varsigma)$ lo que es se muestra, primigeniamente, como real. El $\tau$ ò ồ, lo que es, está siendo real, y porque se nos aparece de modo real es que podemos saber que es. ${ }^{12}$ La realidad del $\tau$ ò ôv , en cualquiera de sus modos, se muestra en tanto que realidad cuando estamos en él, pero si es verdad que el estar patentiza el carácter de realidad de lo que es, entonces considero que los muchos modos en que se dice que es ( $\pi \mathrm{o} \lambda \lambda \alpha \chi \tilde{\omega} \varsigma$ $\lambda \varepsilon$ $\gamma \varepsilon \tau \alpha$ iò òv) devienen muchos precisamente por los muchos modos en los que están presentes las realidades en tanto que realidades. Esto es: porque la realidad se constituye de varios grados, lo que es se dice de muchos modos. El modo como la fuerza está, el modo como es real, difiere modalmente de como lo está Dios, pues uno está (es real) como causa física, mientras que el otro lo está (es real) como causa metafisica. Esta diferencia modal es una diferencia no de naturaleza, sino tan sólo de grado. De naturaleza no, porque de algún modo ambos son reales, pero sí de grado porque ese "algún modo" en que son reales no es el mismo modo. Si lo que es se dice de muchas maneras es porque la realidad se constituye de muchos grados.

Al estar graduada, podemos entonces decir que la realidad posee estructura.$^{13}$ Hablar de grados nos permite distinguir, pues, entre la piedra,

\footnotetext{
${ }^{10}$ Met. 1026a33-b2 y 1028a10-13. Tò ôv es algo difícil de traducir, se dice respecto de عĩval como tò

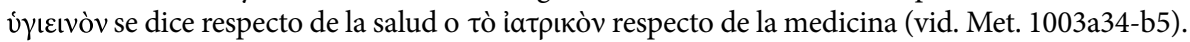
Sin embargo, no es labor de este texto entrar en la discusión de lo que sea tò ôv, por ello simplemente nos referiremos a ello no como traducción sino como denotación con "que es".

${ }^{11} \mathrm{El}$ adverbio $\pi \mathrm{o} \lambda \lambda \alpha \chi \tilde{\omega} \varsigma$ me permite hablar de modos.

${ }^{12}$ Incluso luego abstraer y preguntar por el puro ser.

${ }^{13}$ En otro lado he tratado el concepto de realidad estructural, coincidiendo en dicho sitio con mucho de lo dicho en este texto; es natural la coincidencia, pues una realidad gradual implica una realidad estructural. Éste texto y el otro trabajan sobre una idea casi idéntica. Vid. SIERRA,
} 
el molusco, la bacteria, el número $\pi$, la supernova, el hombre (también los ángeles, si se quiere,) y Dios, sin por ello caer en dualismos o pluralismos que escindan esquizoidemente la realidad; pues, aunque grados, son grados de realidad. Grados de una realidad estructurada, como muchos sabemos, sugeridos ya por Dionisio Areopagita y explicitados por el mismo Santo Tomás de Aquino. ${ }^{14}$ Puesto que la realidad posee estructura, nos es posible hablar de manera coherente matemática, física, química, biológica, artística, filosófica y teológicamente. Los modos como se dicen los grados estructurales de la realidad son muchos, y dependen del modo como están. Según como lo esté, la realidad se dice de muchos modos. Sin embargo, con el riesgo de parecer reduccionistas pero sin serlo, proponemos tres a partir de los cuales se podrían deducir muchos otros o a los cuales podrían pertenecer muchos más; estos tres modos como se dicen los grados de realidad según el modo como están, son: la consistencia, la existencia y la subsistencia. El grado de realidad difiere entre el Último Teorema de Fermat, la Primera Ley de Kepler y la Res Divina, esto es, entre conceptos (matemáticos o no), entre objetos físicos (i.e. materiales) y realidades metafísicas (religiosas o no).$^{15}$ Existen graves confusiones en los grados de realidad cada vez que se intenta investigar a uno con herramientas diseñadas para otros grados, no debe hablarse de cosas diferentes con los mismos términos, no debería buscarse $\mathrm{X}$ con herramientas diseñadas para Y. Sin embargo, aunque no se debería, se hace; y es causa de confusión. Estas confusiones son inflexiones entre grados que, las más de las veces, son peligrosas. Ha ocurrido, por dar un ejemplo, que quiere tratarse de Dios matemática o físicamente, saltando gratuitamente de resultados

C. ¿Por qué decimos que la Realidad es una y que, además, tiene ventanas distintas? In. C. Navarro y G. Chamorro (eds.), Ciencia y Fe: dos ventanas una realidad. Guatemala: Sociedad Educativa Latinoamericanapara Fe y Ciencia, 2013, pp. 23-37.

${ }^{14}$ El concepto de realidad gradual lo considero necesarísimo para un discurso coherente capaz de integrar los grados del saber según los grados de realidad; y sin embargo, aunque la resalto - pues hace falta subrayar que no se puede hablar con los instrumentos de un grado sobre otros grados distintos-, no es un idea novedosa. Como he mencionado, los grados de realidad han sido sugeridos al menos en lo tocante a lo teológico como jerarquía celestial ya por Dionisio Areopagita (Cfr. DIONISIO. La jerarquía celeste. In: Martin-Lunas, T. H. (ed), Obras completas del Pseudo Dionisio Areopagita. Madrid: BAC, 1990, pp. 119-188) y por su lado, ya reconocía explícitamente estos grados el Doctor Angelicus (Cfr. AQUINO, T. Suma contra los gentiles, México: Porrúa: 2004, p.647, 648. [Libro IV, Capítulo XI, al inicio]). Una realidad gradual, aunque no es una idea nueva, sí que es urgente.

${ }^{15}$ Claro que la Primera Ley de Kepler no es un objeto físico, pero con ella se está diciendo simbólicamente lo que en principio es algo físico: el movimiento de los planetas (y que describimos conceptualmente como trayectorias elípticas). 
científicos a conclusiones metafísicas. Spinoza creyó poder hablar de Dios con un método geométrico, hoy no son pocos los físicos que desde la ciencia física en tanto que ciencia física buscan desacreditar aseveraciones religiosas, y tampoco son pocos los religiosos quienes buscan sustentar sus aseveraciones de fe desde aseveraciones físicas. A mi parecer, es igualmente grave el que se quiera desacreditar una proposición de fe desde la ciencia como el que se quiera sustentarla, una cosa es apoyar la hipótesis de un grado con tesis de otros grados, ${ }^{16}$ pero otra es querer fundamentar de la misma manera como se fundamentaría en otros grados, y esto es peligroso. ¿No es esto notable cada vez que con la Biblia se quieren dar explicaciones físicas del mundo? ¿No debería ser igualmente notable cada vez que con las explicaciones físicas del mundo se quieran sustentar los contenidos bíblicos? La inflexión es la misma, y quizá mucho haya sido causado por una palabra que ha perdido gran parte de su concepto (a pesar o debido a la basta especulación filosófica al respecto); tal palabra fuente de confusiones es, a saber, "existencia". Sin entrar en detalles de lo que nos significa el existir, proponemos por ahora operacionalmente -ya habrá tiempo de clarificar las nociones en algún trabajo ulterior- tres modos en que se dicen estructuralmente los grados constitutivos de la realidad, estos son: para la "existencia" conceptual decimos más bien consistencia; para la llana "existencia" física, existencia sin más; y para la "existencia" metafísica, subsistencia. Así pretendemos acotar esa palabra difusa fuente de debates ociosos. Lo real conceptual consiste, lo real físico existe y lo real metafísico subsiste. El esquema de la pregunta por la verdad en cada caso es: ¿es este concepto consistente con $\mathrm{X}$ ? ¿Es esto físico verdaderamente existente? ¿Es verdad que subsiste $\mathrm{X}$ ente metafísico? Y en su respuesta podrá distinguirse claramente el acercamiento requerido: si matemático, lógico, físico, científico, metafísico, filosófico, teológico, religioso, etc.

\section{La Resistencia de la Realidad}

Todo es igualmente real en tanto que real, pero no es igualmente real en tanto el modo como lo está. Los conceptos son en algún modo bien reales, baste con intentar pensar no-occidentalmente estando inmiscuido en la historicidad occidental para notar que la propiedad real de los conceptos se resiste a la propia realidad histórico-cultural humana, por ejemplo, nos es

\footnotetext{
${ }^{16}$ Por ejemplo, para hacer plausible algo (verosímil), mas no para hacerlo posible (verdadero).
} 
muy difícil como occidentales asumir el concepto de un tiempo cíclico en vez de uno lineal, sin embargo, no decimos que los conceptos existen, sino que consisten (el concepto de tiempo lineal es consistente con una historicidad occidental); esto es, los conceptos son consistentes o no (o indecidibles, como pensaría Gödel para conceptos lógico-matemáticos $)^{17}$ según un determinado marco conceptual. El cuatro no existe como la suma de dos más dos, sino que consiste; es consistente como la suma de determinados números según las reglas y la lógica de, por ejemplo, la aritmética de Peano. ${ }^{18}$ Por su parte, los objetos físicos son a su modo reales, cualquiera que haya caído y tropezado habrá atestiguado la propiedad real de lo que llamamos gravedad ${ }^{19}$; decimos de los objetos que existen, que existen físicamente. Por último, las realidades transcendentes son también muy reales, por ejemplo la oủoía - como patrimonio que permite la independencia de una determinada cosa y que por lo tanto se le ha de llamar, luego, substancia - es real, pues sin este patrimonio substancial la cosa, justamente, no subsistiría; por ejemplo, el mundo como un todo - en tanto que es pensado como una substancia unitaria- no subsistiría como un todo si no fuera por esa su unidad; así pues, decimos que la totalidad qua totalidad no existe sino que subsiste.

Pero estos tres modos como decimos lo real - la consistencia, la existencia y la subsistencia - ¿de dónde provienen? ¿Por qué son modos distintos entre sí? No podemos decir cualquier cosa de nada, yo no puedo decir sin más que un número entero elevado a una potencia mayor que dos puede expresarse como

17 Vid. GÖDEL, K. Sobre sentencias formalmente indecidibles de Principia Mathematica y sistemas afines. In. J. Mosterín (ed.), Obras Completas. Madrid: Alianza, 2006, pp. 53-87. Particularmente los teoremas VI para la indecibilidad y XI para la imposibilidad de probar la propia consistencia.

${ }^{18}$ Que obedece a los siguientes axiomas:

1. $1 \in \mathbf{N}$.

2. $a \in N . J . a=a$.

3. $a, b, c \in N . J: a=b .=. b=a$.

4. $a, b \in \mathbf{N} . J \therefore a=b . b=c: J . a=c$.

5. $a=b . b \in \mathbf{N}: \supset . a \in \mathbf{N}$.

6. $a \in \mathbf{N}$. . . $a+1 \in \mathbf{N}$.

7. $a, b \in \mathbf{N} . J: a=b .=. a+1=b+1$.

8. $a \in \mathbf{N} . J . a+1-=1$.

9. $\mathrm{k} \in \mathbf{K} . \therefore 1 \in \mathrm{k}: \mathrm{x} \in \mathbf{N} . \mathrm{x} \in \mathrm{k}:$ J $\mathrm{x} . \mathrm{x}+1 \in \mathrm{k}::$ J . N $\supset \mathrm{k}$.

Cfr. PEANO, I. Arithmetices Principia, nova método expósita. Romae: Ediderunt Fratres Bocca, 1889. p. 1.

${ }^{19}$ Y que conceptualmente cuantificamos con la conocida ecuación de Newton: $\boldsymbol{F}=G\left[\left(m_{1} m_{2}\right) / r^{2}\right]$ 
la suma de dos enteros elevados a esa misma potencia, ${ }^{20}$ como tampoco decir llanamente que lo que ocurre en un punto del espacio influye inmediatamente en otro punto cualquiera, ${ }^{21}$ menos aún que la nada posee entidad alguna. ${ }^{22}$ Sabemos de lo real porque principalmente se nos resiste. Es decir, una inteligencia humana, ante la cual las realidades aparecen como realidades, no puede aseverar nada que las realidades mismas no le permitan. Y son las realidades en cuanto tales las que se resisten a que la inteligencia piense de ellas cualquier cosa, tal resistencia da muestra del carácter real de la realidad. ${ }^{23}$ Uno se percata de que algo es real no tanto por la coherencia del algo como por su resistencia. La resistencia, pues, es quien debe decirme cómo puedo investigar y cómo no, cómo debo acercarme a lo real.

\section{El Principio de Correspondencia Ontológico-epistemológico}

El modo de acercamiento debe ser viable sólo en función del grado de realidad; pues son bien distintos - y así deben mantenerse - los acercamientos matemáticos o experimentales de los míticos o rituales; aunque, como es sabido, es posible utilizar por analogía modos de acercamiento diversos, mas tengamos claro que este préstamo de herramientas es sólo una analogía, no un traspaso a ultranza de herramientas. No busquemos a X con herramientas diseñadas para Y. La jurisdicción de los juicios de nuestras investigaciones la

${ }^{20} x^{n}+y^{n} \neq z^{n} \forall(x, y, z) \in \mathbb{Z} \quad$ y $\forall n>2$. Este es el llamado Último Teorema de Fermat (Cubum autem in duos cubos, aut quadratoquadratum in duos quadratoquadratos, et generaliter nullam in infinitum ultra quadratum potestatem in duos eiusdem nominis fas est dividere cuius rei demonstrationem mirabilem sane detexi. Hanc marginis exiguitas non caperet). Para su demostración, vid. WILES, A. Modular elliptic curves and Fermat's Last Theorem. Annals of Mathemathics 142 (1995), 443-551.

${ }^{21}$ Pues violaría lo deducido del segundo principio de la Relatividad Especial. "Todo rayo luminoso se mueve en el sistema de coordenadas "de reposo" con una velocidad fija $V$, independientemente de si este rayo luminoso sea emitido por un cuerpo en reposo o en movimiento." EINSTEIN, A. Sobre la electrodinámica de cuerpos en movimiento. In. S. Hawking, (ed.). A hombres de gigantes. Barcelona: Crítica, 2010, pp. 1027-1052.

${ }^{22}$ Porque si la nada es, es algo y no ya nada. "La nada es la completa negación de la totalidad de lo ente" HEIDEGGER, M. ¿Qué es metafísica?. In. Heidegger, M. Hitos. Madrid: Alianza, 2007, p.93-108.

${ }^{23} \mathrm{El}$ enjuiciamiento de esa realidad resistente es un interesante tema pero aparte. Uno puede enjuiciar, quizá, lo que sea de la realidad, y tal enjuiciamiento mucho tendrá que ver con la precomprensión del todo desde la que inevitablemente partimos cada cual; pero sea cual sea el enjuiciamiento, será el carácter resistente de la realidad el que por convergencia haga del juicio - sea afirmativo o negativo - uno verdadero o falso. 
da la propia realidad investigada; y el uso analógico de las herramientas sólo es legítimo en tanto analógico. Por esta razón, la matemática puede compartir sus métodos de inferencia estadística, por ejemplo, con la sociología; pero no así la física compartir sus ecuaciones diferenciales con las disertaciones exegéticas en torno al Génesis (incluso, no pueden siquiera compartirse a secas - los métodos de las exégesis cristianas del Génesis con los de las

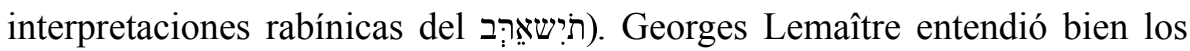
grados de realidad, no confundiendo lo que luego habría de llamarse Big Bang con la Creación. ${ }^{24}$ Pueden compartirse las herramientas -como les he dicho a los métodos, lógicas, conceptos, etc.-, pues al fin y al cabo la realidad aunque con grados - sigue siendo una, pero no olvidemos su resistencia.

Es posible, pues, un préstamo analógico de herramientas conducido por el modo en que se resisten los grados de realidad, pero no una transposición de los métodos ignorando toda gradación real o, más grave aún, jerarquizando las gradaciones, como si unas legitimasen otras, como si, por ejemplo, lo físico legitimara lo metafísico y sólo hasta entonces lo metafísico cobrara algún valor. Lo metafísico debe adquirir valor desde sí mismo, y si bien echa mano de lo físico, eso físico no es lo que fundamentalmente dota de valor a lo metafísico. Considero que no es que un grado de realidad sea consecuencia de otro, y por ello se legitime transferir a ultranza los resultados de un grado a las conclusiones de otros; sino más bien que un grado de realidad es complementario con el resto de grados. No es lo mismo la consecuencia de

\footnotetext{
24 "Y si ese sacerdote [Lamaître] proponía, además, que el universo tenía un origen histórico, la presunta intromisión parecía confirmarse: se trataría de un sacerdote que quería meter en la ciencia la creación divina. Pero los trabajos científicos de Lemaître eran serios, y finalmente todos los científicos, Einstein incluido, lo reconocieron y le otorgaron todo tipo de honores. Lamaître jamás intentó explotar la ciencia en beneficio de la religión. Estaba convencido de que ciencia y religión son dos caminos diferentes y complementarios que convergen en la verdad." Op. Cit. Ciencia y fe: el origen del universo, Georges Lemaître: el padre del big-bang. Sin embargo, es posible que parezca que el Papa Pio XII sí confundió los grados de realidad de los que se habla en el Big Bang y el Génesis: "[Physical proofs have] confirmed the contingency of the universo [...] Hence creation took place in time. Therefore, there is a Creator. Therefore, God exists!", citado por CARROLL, W. Cration in the Age of Modern Science. Tópicos 42 (2012) 107-124. Existen todavía hoy, sin embargo y de manera lamentable, físicos eminentes que confunden estos grados de realidad. Para una excelente señalización de que las ciencias no pueden concluir metafísicamente en tanto que ciencias, sino sólo tendiendo puentes con la filosofía y/o la teología, es decir, en tanto que ya no son puramente ciencias, véase: ARTIGAS, M. Dios y la ciencia. Jean Guitton dialoga con los científicos, Ciencia, razón y fe, Universidad de Navarra, junio 1993. Disponible en: <http://www.unav.es/cryf/diosylaciencia.html > Consultado el $13 \mathrm{de}$ septiembre de 2012 .
} 
$Q$ a $P$, que la complementariedad entre $P$ y $Q$. La totalidad de lo real es una totalidad estructurada y, por ello (con la salvedad de la particular relación entre Dios y el resto de la totalidad), los grados de realidad no son consecuentes sino más bien constitutivos. Y constitutivos pueden ser nuestros acercamientos a esa realidad si se corresponden con ella. Siempre que tomemos en cuenta la complementariedad y resistencia que esos mismos grados permiten e impiden, entonces la complementariedad de nuestros grados del saber se corresponderá con la complementariedad de los grados de realidad. Así pues, tomando prestado análogamente el Principio de Correspondencia de N. $\mathrm{Bohr},{ }^{25}$ que puede enunciarse someramente como que la física clásica debe de emerger como una aproximación a la física cuántica a medida que los sistemas aumentan de tamaño, ${ }^{26}$ nosotros decimos que tal correspondencia puede entenderse como que nuestros grados del saber emergen como una aproximación a otros grados del saber en la medida en que cambiamos de grado de realidad. No es válido permanecer en un grado del saber cuando cambiamos de grado de realidad. Enunciemos formalmente el principio de correspondencia ontológico-epistemológico:

Si el grado de realidad va cambiando en el acercamiento a ésta, entonces deberán emerger correspondientemente otros grados del saber.

Yo puedo acercarme al objeto X con mi herramienta $\mathrm{Y}$, tanto como $\mathrm{X}$ se deje corresponder por $\mathrm{Y}$, y si no se deja, habrá que cambiar a la herramienta Z. Los grados de nuestro saber se corresponden con los grados de realidad siempre que sepamos que éstos se constituyen entre sí a la vez que se nos resisten. He aquí un ejemplo de esta correspondencia resistida: cuando el matemático Cauchy nombra infinito al límite de los "valores numéricos sucesivos de una misma variable que crecen más y más de modo que rebasan cualquier número dado"27, no podemos sino recordar el afamado id quo maius

\footnotetext{
${ }^{25}$ Vid. HEISENBERG, W. Física y filosofía. Buenos Aires: La Isla, 1959.

${ }^{26}$ El principio de correspondencia está íntimamente ligado a la constante de Planck, $h$. Cuando, en un sistema físico, $h$ tiende a cero, entonces el valor de $h$ (que en sí mismo no es cero) se torna despreciable, y podemos mantenernos en un sistema físico clásico. Si $h$ no tiende a cero, entonces su valor, que no es nulo, es decisivo para el sistema, así que nos encontramos en uno cuántico.

${ }^{27}$ De su Cours d'Analyse, citado por Jan Sebestik en la Introducción al libro de Bolzano Paradoxien Des Unendlichen, In: BOLZANO, Bernard, Las paradojas del infinito, México: Mathema, 2010, pp. VI, VII.
} 
cogitari non potest de San Anselmo, ${ }^{28}$ además de remitirnos a San Agustín quien, pensando en el infinito matemático como cualquier número mayor a cualquiera imaginable, tacha de impíos a quienes no aceptan que Dios pueda conocer todos los números ${ }^{29}$; quizá por ello, el matemático y teólogo Bolzano utilizase análogamente su noción de infinito teológica para abordar su noción de infinito matemática; ${ }^{30}$ invitación teológico-matemática que remite a la teológica-política de Spinoza $^{31}$, la cual nos recuerda a la lógica-filosófica de Wittgenstein ${ }^{32}$; retomados en los números transfinitos de Cantor, ${ }^{33}$ los infinitos que son a la vez infinitos del propio Bolzano, parecen tener relación con la propuesta metafísico-panenteista del triple infinito spinozista; ${ }^{34}$ propuesta de Spinoza que a la vez es tomada de su tradición judaica en la que con fuerza se repite שודק שודק שודק, cuando Isaías es llevado a donde Dios (Is. 6:3); que también, por su parte, fue apocalípticamente cristianizada en el trisagio, cuando San Juan es asimismo llevado frente Dios (Ap. 4:8), triple santo interpretado teológicamente como una señal de la Trinidad, etc.

Este ejemplo de correspondencia resistida es muestra de una red constitutiva de correspondencias que llegan hasta donde las resistencias les permiten, no más. Un grado puede ser el motus de la investigación de otro grado, aquí lo ejemplificamos conectando de Cauchy a la Trinidad, esto es, de la matemática a la teología, pero el que sea el motus no implica que sea su regula. La correspondencia en los grados del saber ${ }^{35}$ sólo es legítima si se aproxima a los grados de realidad, ${ }^{36}$ pues los grados de realidad deben mantenerse como grados

\footnotetext{
${ }^{28}$ SAN ANSELMO, Proslogium. The latin library. Disponible en: <http://www.thelatinlibrary. com/anselmproslogion.html> Consultado el 13 de septiembre de 2012. [Capítulos II y XV]

${ }^{29}$ Vid. Libro duodécimo, capítulo XIX de De Civitate Dei. SAN AGUSTÍN, La Ciudad de Dios, México: Porrúa, 2006, pp.334, 335.

${ }^{30}$ Hablando, por lo demás, de las infinitas fuerzas de Dios cada una de las cuales es también infinita, como lo son un conocimiento infinito, una verdadera omnisciencia y un conjunto infinito de verdades Vid. $\$ 11$ de Op. Cit. Las paradojas del infinito, pp.17, 18.

${ }^{31}$ Vid. SPINOZA, B. Tratado teológico-político, Madrid: Tecnos, $1996 .$.

${ }^{32}$ Vid. WITTGENSTEIN, L. Tractatus logico-philosophicus. Madrid: Alianza, 2002.

${ }^{33}$ Vid. CANTOR, G. Fundamentos de la teoría de los números transfinitos, In. S. Hawking (ed.). Dios creó los números, Barcelona: Crítica, 2010. Y CANTOR, G. Contributions to the founding of the theory of transfinite numbers, México: IPN, 1999.

${ }^{34}$ Siendo Dios un infinito con infinitos atributos cada uno de los cuales es también infinito. Vid. Primera parte, definición VI de SPINOZA, B. Ética demostrada según el orden geométrico, La Plata: Terramar, 2005, p.17.

${ }^{35}$ Vid. MARITAIN, J. Distinguir para unir o los grados del saber, Argentina: Ediciones DDB, 1947.

${ }^{36}$ Lo que digamos en los grados del saber sólo es verdad según el grado de realidad. Aunque no
} 
en función de una resistencia bien específica y manifiesta en el estar; presente siempre que se está en cada grado que es - en sentido fuerte - real. Confundir los grados de realidad me parece inadmisible, porque existe no sólo una inflexión de grados sino también una tergiversación cuando por la regula de un grado del saber se quiere hacer pasar cualquier grado de realidad. Es como querer encontrar al homo neanderthalensis en la Biblia y, al no encontrarlo, afirmar de Ella ser un libro equivocado, cuando ocurre justo a la inversa: soy yo, que soy quien busca, quien se equivoca por no buscar en el libro correspondiente, uno de paleontología: no es el libro quien se equivoca, sino yo que me equivoco de libro. Hay modos de realidad trascendentes y los hay inmanentes, hay consistencias, existencias y subsistencias. Y el modo cómo consistir, existir y subsistir queda específicamente tipificado en la resistencia patente en que el algo consiste, existe o subsiste. De hecho, el modo de justificación que se exige en cada cual deberá ser también diferente: en la consistencia, una consecuencia lógica; en la existencia, una evidencia empírica; en la subsistencia, una sistematización filosófica o teológica ${ }^{37}$, o si es subsistencia religiosa, se necesitará una vivencia. ${ }^{38}$ Quizá esto bien claro lo tenían el Cardenal Baronio, Galileo ${ }^{39}$ y Juan Pablo II al decir que las Sagradas Escrituras fueron escritas para mostrarnos cómo llegar a los cielos, no para mostrarnos cómo funcionan. ${ }^{40}$

\section{Conclusión}

Nuestro decir sobre los grados debe corresponder a los propios grados. Si Dios subsiste es que no existe como objeto físico - aunque en ellos pueda tener determinadas manifestaciones -, entonces no usemos herramientas propias de las realidades existentes para la realidades subsistentes, tanto más cuando ésta

sólo en ese grado Esto es: la verdad matemática $2+2=4$ es tal según los estándares de la aritmética, pero es verdadera incluso para Dios, aunque con ella no hagamos argumentación teológica alguna. Por su parte, la verdad de los dogmas cristianos es tal según los dogmas mismos, por lo que, aun cuando sean verdad, no argumentaremos con ellos el que $2+2=4$.

${ }^{37}$ Que debe atenerse a la lógica y a la empírea pero no detenerse en ellas.

${ }^{38}$ Claro que el modo de justificar las subsistencias es mucho más complicado, pero en principio sería como se señala en el texto.

${ }^{39}$ Cfr. WRIGHT, E.L., Cosmology and Religion. Division of Astronomy and Astrophysics (UCLA), California, noviembre de 2010. Disponible en $<$ http://www.astro.ucla.edu/ $\sim$ wright/cosmo-religion.html> Consultado el 13 de septiembre de 2012.

40 "Spiritui Sancto mentem fuisse nos docere quomodo ad coelum eatur, non quomodo coelum gradiatur." (La intención del Espíritu Santo era enseñarnos cómo ir al cielo, no cómo tomarle medidas) Vid. O’COLLINS, G (ed.). Pope John Paul II: a reader. New Jersey: Paulist Press, 2007, p. 33. 
se trata de un Dios qui est Ipsum Esse per se Subsistens. Así tampoco con los argumentos a priori, K. Gödel trabajó en un argumento ontológico, más no lo publicaba por miedo a que pensaran que quería demostrar la «existencia» de Dios cuando lo único que hacía era demostrar la consistencia de una determinada proposición con un determinado sistema conceptual regido por un determinado juego de reglas lógicas. ${ }^{41}$ Nada más. No confundamos la consistencia de los conceptos, ideas o representaciones mentales, ni con la existencia de los objetos dados a la intuición sensible, a la empírea, a la experiencia, ni con la subsistencia de los sujetos metafísicos, trascendentes, aquellos que exceden la mera representación y la mera sensación. Aquello con lo que la inteligencia contacta, que se pone de manifiesto ante ella como siendo real, se hace presente de maneras muy precisas y distintas; es ello, en su carácter resistente, el que debe guiar nuestras investigación señalando hasta dónde podemos decir de ellos y con qué clase de acercamientos. ¿Cómo escudriñar la realidad? En función de cómo la realidad se haga presente, esto es, según como esté yo con las cosas y cómo lo estén ellas conmigo. Es al atestiguamiento de la resistencia de lo real lo que debemos atender a la hora de operar según el principio de correspondencia ontológico-epistemológico, sólo en esa medida no defraudaremos al contenido efectivo de lo contactado en los actos de intelección, en el percipere; asimismo, nuestros discursos tendrán un base que los unifique a pesar de aparentes contradicciones entre ellos (sean los que sean), pues distinguiendo lo percipi, habrá claridad para el percipiens.

Con los grados del saber complementándose entre sí y correspondiéndose con los grados de realidad que a su vez se complementan entre sí, podemos generar un discurso coherente sin reduccionismos, integral y consciente de las diversas dimensiones humanas que por ningún motivo deben aniquilarse. Porque los grados de la totalidad de lo real no se excluyen sino que se constituyen, la realidad es un todo complejo que da cobijo a todo tipo de apreciaciones y preocupaciones humanas, y no sólo a unas cuantas «únicas» capaces de legitimar al resto. La realidad no es mezquina, procura la integridad de toda fase que la constituya porque quizá lo que en el fondo procura sea la fase propia de la realidad humana.

${ }^{41}$ Cfr. PARK, W. On the motivation of Goedel's ontological proof. The Modern Schoolman 80 (2003) 144-153. 


\section{Referencias Bibliográficas}

AQUINO, Tomás de. Suma contra los gentiles. México: Porrúa, 2004.

AQUINO, Tomás de. De ente et essentia. Luventicus, 9 de diciembre de 2003. Disponible en $<$ http://www.luventicus.org/articulos/03Tr002/index. html\#IIb>. Consultado el 13 de septiembre de 2012.

ARISTÓTELES, Metafísica. Madrid: Gredos, 2008.

ARISTÓTELES, Aristotle's Metaphysics, Oxford: Clarendon Press (ed. W.D. Ross), 1924.

ARTIGAS, Mariano. Ciencia y fe: el origen del universo, Georges Lemaitre: el padre del big-bang. Ciencia, razón y fe, Universidad de Navarra, 7 junio 1995. Disponible en: <http://www.unav.es/ cryf/georgeslemaitreelpadredelbigbang.html>. Consultado el 24 de septiembre de 2012.

ARTIGAS, Mariano. Dios y la ciencia. Jean Guitton dialoga con los científicos, Ciencia, razón y fe, Universidad de Navarra, junio 1993. Disponible en: <http://www.unav.es/cryf/diosylaciencia.html> Consultado el 13 de septiembre de 2012.

BOLZANO, Bernard. Las paradojas del infinito. México: Mathema, 2010.

CANTOR, Georg. Fundamentos de la teoría de los números transfinitos. In. Hawking, Stephen, Dios creó los números. Barcelona: Crítica, 2010.

CANTOR, Georg. Contributions to the founding of the theory of transfinite numbers. México: IPN, 1999.

CAROLL, William. Cration in the Age of Modern Science. Tópicos 42 (2012) 107-124.

DIONISIO. La jerarquía celeste. In: Martin-Lunas, T. H.(ed), Obras completas del Pseudo Dionisio Areopagita. Madrid: BAC, 1990, pp. 119-188

EINSTEIN, Albert. Sobre la electrodinámica de cuerpos en movimiento. In.

S. Hawking, (ed.). A hombres de gigantes. Barcelona: Crítica, 2010, pp. 1027-1052.

GÖDEL, Kurt. Sobre sentencias formalmente indecidibles de Principia Mathematica y sistemas afines, In: J. Mosterín (ed.), Obras Completas. Madrid: Alianza, 2006, pp. 53-87. 
HEIDEGGER, Martin. ¿Qué es metafísica?. In. Heidegger, M. Hitos. Madrid: Alianza, 2007, p.93-108.

HEISENBERG, Werner. Física y filosofía. Buenos Aires: La Isla, 1959.

MARITAIN, Jacques. Distinguir para unir o los grados del saber. Argentina: Ediciones DDB, 1947.

O'COLLINS, Gerald (ed.). Pope John Paul II: a reader. New Jersey: Paulist Press, 2007.

PEANO, Ioseph. Arithmetices Principia, nova método expósita. Romae: Ediderunt Fratres Bocca, 1889.

SAN AGUNTÍN. La Ciudad de Dios. México: Porrúa, 2006.

SAN ANSELMO. Proslogium. The latin library. Disponible en: <http:// www.thelatinlibrary.com/anselmproslogion.html $>$ Consultado el 13 de septiembre de 2012.

SIERRA, Carlos. ¿Por qué decimos que la Realidad es una y que, además, tiene ventanas distintas? In. C. Navarro y G. Chamorro (eds.), Ciencia y $\mathrm{Fe}$ : dos ventanas una realidad. Guatemala: Sociedad Educativa Latinoamericanapara Fe y Ciencia, 2013, pp. 23-37.

SPINOZA, Baruch. Ética demostrada según el orden geométrico. La Plata: Terramar, 2005.

SPINOZA, Baruch. Tratado teológico-político. Madrid: Tecnos, 1996.

WILES, A. Modular elliptic curves and Fermat's Last Theorem. Annals of Mathemathics 142 (1995), 443-551.

WITTGENSTEIN, Ludwug. Tractatus Logico-philosophicus. Madrid: Alianza, 2002.

WRIGHT, E.L. Cosmology and Religion. Division of Astronomy and Astrophysics (UCLA), California, noviembre de 2010. Disponible en $<$ http://www.astro.ucla.edu/ wright/cosmo-religion.html $>$ Consultado el 13 de septiembre de 2012.

ZUBIRI, Xavier. El hombre: lo real y lo irreal. Madrid: Alianza, 2005.

ZUBIRI, Xavier. El hombre y la verdad. Madrid: Alianza, 2005. 
ZUBIRI, Xavier. Inteligencia sentiente: inteligencia y realidad. Madrid: Alianza, 1980.

ZUBIRI, Xavier. Los problemas fundamentales de la metafisica occidental. Madrid: Alianza, 2008.

ZUBIRI, Xavier. Reflexiones teológicas sobre la eucaristía. Estudios escolásticos 56 (1981) Enero-Junio, 41-59.

ZUBIRI, Xavier. Respectividad de lo real. Realitas III-IV (1976-1979), 13-43.

ZUBIRI, Xavier. ¿Qué es investigar? The Xavier Zubiri Review 7 (2005), 5-7.

Carlos Sierra Lechuga

Licenciado en Filosofía por la Universidad del Claustro de Sor Juana (UCSJ), México Miembro de la Sociedad Educativa Latinoamericana para Fe y Ciencia (SELFYC)

Miembro del grupo de traductores del Dr. Herwig Weber (UCSJ) Vicepresidente Asociado Fundador de la Academia Internacional Tomás de Aquino (AITA) Miembro del Círculo de Filosofía de la Naturaleza Université de Strasbourg, França E-mail: rideo_ergo_sum@hotmail.com

Recebido em 05/12/12 Aprovado em 11/03/13 